\title{
CRYSTALLIZATION KINETICS STUDY OF IMPACT POLYPROPYLENE COPOLYMER WITH KENAF AS NUCLEATING AGENT AND REINFORCEMENT
}

\author{
Jaka Fajar Fatriansyah ${ }^{1 *}$, Muhammad Joshua Yuriansyah Barmaki ${ }^{1}$, Rahma Lailani ${ }^{1}$, \\ Mochamad Chalid ${ }^{1}$ \\ ${ }^{1}$ Department of Metallurgical and Materials Engineering, Faculty of Engineering, Universitas \\ Indonesia, Kampus UI Depok, Depok 16424, Indonesia
}

(Received: August 2018 / Revised: March 2019 / Accepted: October 2019)

\begin{abstract}
Impact Polypropylene Copolymer (IPC) is an material which combines properties of isotactic Polypropylene (iPP) and its own high impact toughness. However, the crystallization kinetics might be lower than iPP, which may affect the manufacturing cycles. Kenaf was used as the addition in IPC, in which kenaf acts as a nucleating agent as well as reinforcement for IPC. Kenaf was alkalized with $\mathrm{NaOH} 6$ v.v\% for about 8 hours to remove the dirt on the surface and to reduce lignin, which contributed polarity to kenaf. The alkalization improved the compatibility of kenaf fiber with IPC. The crystallization kinetics study was conducted by employing the Avrami model to Differential scanning calorimetry data in order to obtain halftime, Avrami index (morphology parameter) and Avrami crystallization kinetics. The addition of $5 \mathrm{wt} . \%$ kenaf was found to be an optimum concentration to improve crystallization kinetics. The addition of more than $5 \mathrm{wt} . \%$ kenaf (15 wt.\% and $20 \mathrm{wt} . \%)$ did not improve crystallization kinetics which may be due to the agglomeration, thus preventing efficient heat transfer between nucleating seeds and the matrix. The connection between crystallization kinetics and mechanical properties was also established for the IPC+ kenaf system.
\end{abstract}

Keywords: Crystallization kinetics; Impact polypropylene copolymer; Kenaf fiber; Nucleating agent; Polymer reinforcement

\section{INTRODUCTION}

Impact Polypropylene Copolymer (IPC) is a unique plastic material, combining properties of isotactic Polypropylene (iPP) which has a good heat resistance (insulator), good toughness (Chen et al., 2009), good manufacturability (Karger-Kocsis et al., 1997), and most important, the superior property of high impact toughness (Michael, 1991). Because of these useful properties, IPC is widely used in many applications such as in packaging, household appliances, automotive parts and even military uses (Hongjun et al., 1999). IPC is commonly produced from homopolymerization of Propylene and followed by copolymerization of propylene and ethylene. Thermal behavior of plastics greatly affects manufacturing process and properties of the product. Thermal behavior is related to the kinetics of crystallization (Chalid et al., 2017; Chalid et al., 2018). Crystallization kinetics of IPC may be lower in comparison with iPP due to the structure of ethylene. This may affect cycle time in the manufacturing process, for example, due to the longer crystallization time. One of the methods to improve the crystallization of kinetic properties is the addition of a nucleating agent (Tolinski, 2009).

*Corresponding author's email: fajar@metal.ui.ac.id, Tel. +62 81283412864

Permalink/DOI: https://doi.org/10.14716/ijtech.v10i5.2267 
Usually natural based fiber is used as a reinforcement to improve mechanical properties of plastic composites due to its advantages such as: low density, availability, degradability as well as less abrasive and good insulator. Natural fibers can be classified into three types: fiber from plants (cellulose or lignocellulose), fiber from animals (protein) and mineral fibers (Akil et al., 2011). Examples of plant based are flax, jute, ramie, kenaf, sisal, bamboo, wheat, maize, barley and sago (Abral et al., 2012). The use of natural based fiber as a nucleating agent in plastics has increasingly attracted some researchers. However, some researchers use natural based fibers as nucleating agents as well. Fundador et. al. (Fundador et al., 2012) used xylan ester from hemicellulose as a nucleating agent for polylactic acid (PLA). Guo et. al. (Guo et al., 2015) used natural protein fiber as a nucleating agent for iPP. They showed that the use of other natural fiber resources as a nucleating agent is possible. Yuanita et al. used Arenga pinnata "ijuk" fiber in PLA nucleating agent (Yuanita et al., 2015) and Prabowo et al. used similar ijuk to modify crystallinity of the iPP composite (Prabowo et al., 2017).

Kenaf (Hibiscus cannabinus), a plant based natural fiber, has high tensile strength (930 MPa) as well as low density (Akil et al., 2011). This high tensile strength makes kenaf suitable as a reinforcement. However, in order to be properly used as a nucleating agent, the interface between kenaf and IPC should be compatible with each other. It is well known that the nonpolar IPC will be incompatible with polar natural fibers: this is the case with kenaf (Spoljaric et al., 2009). One of the methods used to reduce the polarity of kenaf is alkaline treatment (alkalization) (Akhtar et al., 2016). The alkalization process on fiber may reduce the polarity of fiber by removing its lignin which donors the polarity property in fiber. Another study of alkali treatment (alkalization) to improve the capability of ijuk to be compatible for reinforcement and/or nucleating agent in polymer was conducted by Chalid and Prabowo (2015) by using $\mathrm{NaOH}$. They found that the crystallinity, which is somehow related to the interface or compatibility with polymer, of ijuk increases after alkalization. The objective of this study is to study the use of $\mathrm{NaOH}$ alkalized kenaf as a nucleating agent as well as reinforcement for IPC. We expect that kenaf will improve IPC crystallization kinetics, which will be analyzed by means of the Avrami crystallization theory. In addition, in this paper, we attempt to establish the relation between crystallization kinetics and mechanical strength (the use of kenaf as reinforcement) through the experimental results.

\section{THEORY}

In order to establish the crystallization kinetics analyses, a simple yet powerful model should be chosen. In this paper, the Avrami model was chosen because this model satisfies those criteria. Avrami (1939) proposed a model which can picture phenomenon of crystallization in materials. The assumptions used are: spontaneous growth, uniform nucleus forming and isothermal condition. The equation can be written as

$$
\theta(t)=1-\exp \left(-K_{\mathrm{av}} t^{n}\right)
$$

where $\theta(t)$ is relative crystallization, $K_{\text {av }}$ is Avrami crystallization kinetic parameter (not to be confused with crystallization kinetics) and $n$ is the Avrami index. Two latter variables are a characteristic parameter which can quantify the phenomenon of crystallization. According to Mahadevan et al. (1986), $n$ value can be 4, 3, 2 or 1 which is related to the glass-crystal transformation mechanism as follows: $\mathrm{n}=4$ equals to volume nucleation (three-dimensional growth), $\mathrm{n}=3$ equals to two dimensional growths, 2 equals to one dimensional growth and 1 equals to one dimensional internal growth. Zhouzhou et al. (2018) calculated polymer crystal growth dynamics by the Dynamic Monte Carlo (DMC) method and proved that one dimensional crystal growth yields more structured crystal orientation and has crystallinity 
degree in comparison with two or three-dimensional growths. Equation 1 can be written as a linear equation as follows:

$$
\ln [-\ln (1-\theta(t))]=n \ln t+\ln K_{t}
$$

By using Equation 2, relative crystallization versus time or temperature (in logarithmic) could be plotted. The gradient of the plot is the Avrami index and the intersection of the curve with the y-axis shows the Avrami crystallization kinetics.

The relative crystallization can be calculated by the following equation:

$$
\theta(\mathrm{t})=\frac{Q_{t}}{Q_{\infty}}=\frac{\int_{0}^{t} \frac{d H}{d t} d t}{\int_{0}^{\infty} \frac{d H}{d t} d t}
$$

where $Q_{t}$ and $Q_{\infty}$ are the enthalpy produced at time $t$ and infinity (ideally) of cooled material and $\frac{d H}{d t}$ is the rate of enthalpy change. Basically, this equation expresses the ratio of enthalpy produced at time $t$ and the time when the material is (presumably) fully crystallized. The integral basically expresses the area under the curve of the Differential Scanning Calorimetry (DSC) heat-temperature curve.

\section{EXPERIMENTS}

This work concerns the crystallization kinetics of IPC and IPC+ kenaf systems. The experiments were conducted based on thermal analyses employing the Avrami crystallization theory.

\subsection{Materials}

The materials used in this study were IPC pellets and kenaf fibers as the main ingredients. $\mathrm{NaOH} 1 \mathrm{M}$ solution also used for alkalization treatment for the surface of the fibers. Kenaf fibers were obtained from a local supplier in Tangerang Indonesia in July 2015. IPC was supplied by the Chandra Asri Petrochemical Tbk, Indonesia. $\mathrm{NaOH}$ was purchased from Wako Chemical, Japan. All of the materials were used without any further purification.

\subsection{Material Preparations}

First, the kenaf was washed in distilled water to remove dirt and other impurities attached to the surface, and then dried for 1 hour to remove the moisture. The water contained in kenaf increases its polarity and should be mitigated in order to increase compatibility with non-polar IPC. Dried kenaf was then cut into small pieces up to $7 \mathrm{~cm}$ and soaked in $\mathrm{NaOH} 6 \mathrm{v} / \mathrm{v} . \%$ solution in ambient temperature $(298 \mathrm{~K})$. The alkalization was conducted for 8 hours. Then, alkalized kenaf was washed 3 times in distilled water until its $\mathrm{pH}$ reached 7. After washing, kenaf was dried and cut again in the Mixer Formac and then filtered in a 40-mesh sieve. The cleaned kenaf then was ready to be mixed with IPC. IPC was mixed with the kenaf fibers with the composition of $5 \mathrm{wt} . \%, 15 \mathrm{wt} . \%$ and $25 \mathrm{wt} . \%$. The hot melt mixing was conducted by Rheomix with a heater temperature of $170^{\circ} \mathrm{C}$ for 15 minutes to achieve a good dispersion and distribution. The hot melt mixing was used to reduce viscosity and thus prevented the agglomeration of kenaf. The cooled IPC+ kenaf was then ready to be further thermal characterized. 


\subsection{Material Characterizations}

Thermal measurements were conducted by using a Differential Scanning Calorimetry PerkinElmer 6000. To conduct the isothermal analysis, one should assume some crystallization temperatures. From the elevated temperature (above melting temperature $175^{\circ} \mathrm{C}$ ), the material should be rapidly cooled to the assumed temperature. In this experiment we used the cooling rate of $10 \mathrm{~K} / \mathrm{min}$ and assumed crystallization temperature is $90^{\circ} \mathrm{C}$. This procedure should be repeated for different temperatures. However, in this study, one value of the temperature was assumed since our purpose was only to compare the kinetics with and without kenaf addition in IPC. All of the thermal measurements should be conducted after erasing thermal memories of the sample by repeatedly heating and cooling the materials rapidly. In addition, to thermal measurements, in order to confirm whether the dirt on the surface of kenaf was removed, FTIR (Fourier Transform Infrared) observations (Perkin Elmer) and FESEM (Field Emission Scanning Electron Microscope) imaging (FEI Inspect F50) were conducted. All of the characterization was conducted in the Department of Metallurgical and Materials Engineering Universitas Indonesia.

\section{RESULTS AND DISCUSSION}

First, the effect of $\mathrm{NaOH}$ alkalization on kenaf morphology will be discussed. As shown in Figure 1 (FESEM imaging), after alkalization, the dirt on the surface of kenaf was totally removed. The lignin, as shown by white surface, was greatly reduced. This result showed that the alkalization was partially successful.
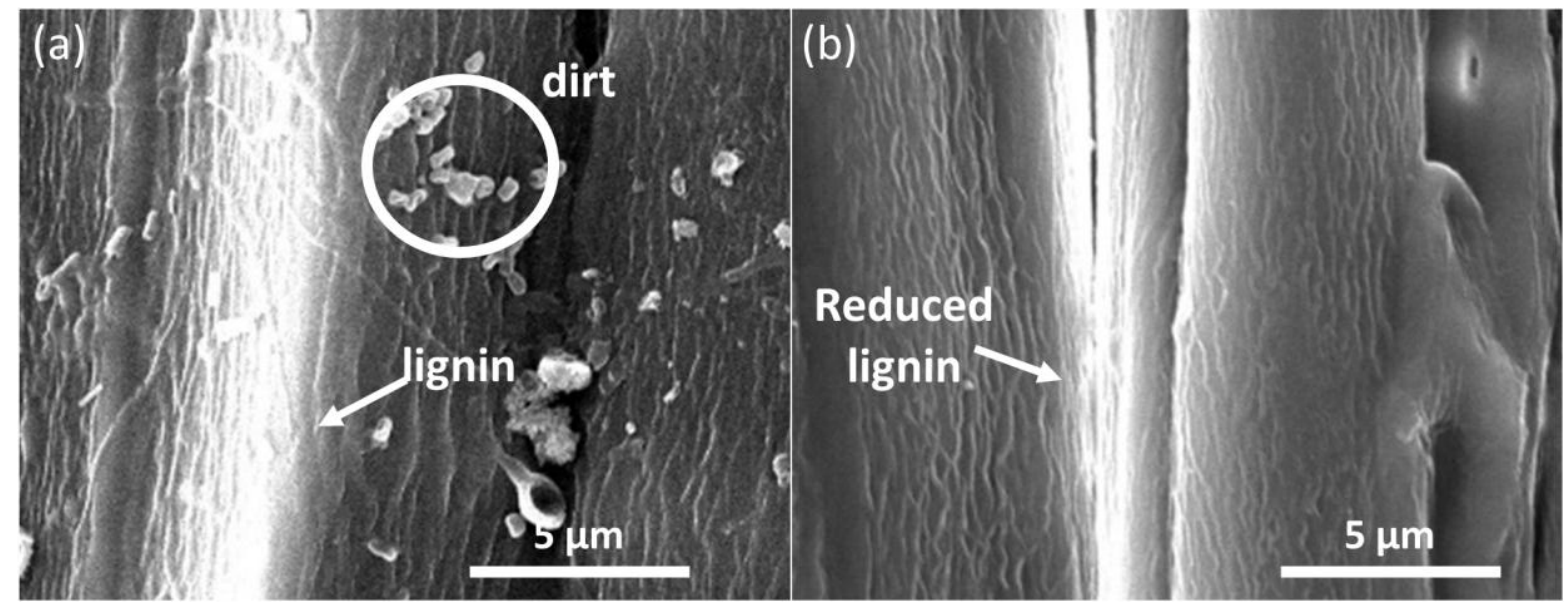

Figure 1 The surface morphology obtained from FESEM imaging of: (a) kenaf; and (b) $\mathrm{NaOH}$ alkalized kenaf. It is clearly shown that the dirt on the surface of kenaf was totally removed and lignin was significantly reduced

Figure 2 shows FTIR spectra for the sample without alkalization and with alkalization. The aromatic ring signal originated from the lignin and hemicellulose $(\mathrm{C}-\mathrm{C}$ stretch in the wavelength of $1421 \mathrm{~cm}^{-1}$ ) was greatly reduced. From this result, the polarity of kenaf was expected to be greatly reduced and thus increasing the compatibility with polymer/IPC. Actually, the polarity of kenaf should be measured by NMR means (chemically) or other compatibility test (physical compatibility, dynamic contact angle) for example. However, from the FTIR observation and FESEM (morphology) results, it is enough to assume that the lignin which brings significant polarity in kenaf was greatly reduced. Thus, the polarity of kenaf was reduced and a good degree of compatibility with IPC should be expected. 


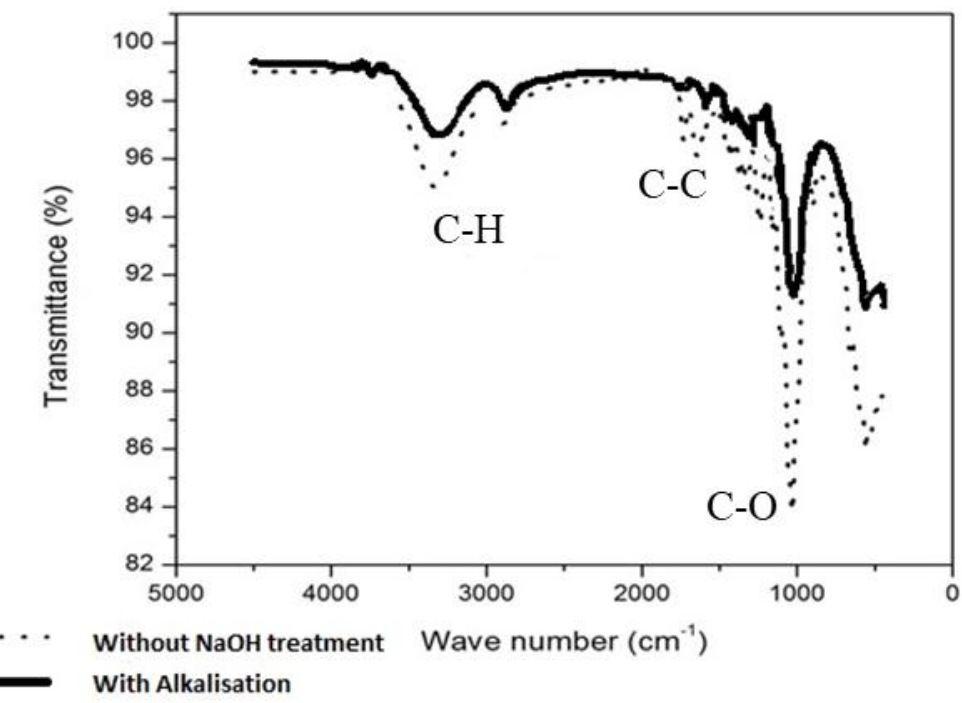

Figure 2 FTIR spectra for the sample without alkalization and with alkalization. The aromatic ring signal originated from the lignin and hemicellulose (C-C stretch in the wavelength of $1421 \mathrm{~cm}-1)$ was greatly reduced

Now, the crystallization kinetics (crystal growth dynamics) of the IPC+ kenaf system will be discussed. Figure 3 shows typical melt crystallization exotherms obtained from DSC for pure IPC, IPC+ 5 wt.\% kenaf, IPC+ 15 wt.\% kenaf and IPC+ 25 wt.\% kenaf. Actually, the experiments should be conducted isothermally. However, since this study was only focused on the comparison of crystallization kinetics parameters in pure IPC and IPC+ kenaf samples, full isothermal calculation is not necessary. It is shown that pure IPC has lower crystallization temperature (shown as peak) at $120^{\circ} \mathrm{C}$ in comparison with IPC $+5 \mathrm{wt} . \%$ kenaf, IPC $+15 \mathrm{wt} . \%$ kenaf and IPC+ 25 wt. $\%$ kenaf which has crystallization temperatures at $128^{\circ} \mathrm{C}, 127.5^{\circ} \mathrm{C}$ and $127^{\circ} \mathrm{C}$, respectively. The increase of crystallization temperature is due to the addition of kenaf which changes the nucleation energy barrier to the sample thus shifting crystallization temperature to the higher temperature. The higher crystallization temperature expects faster crystallization kinetics (for similar heating/cooling rate) since with similar cooling rate, the system releases a lower total energy.

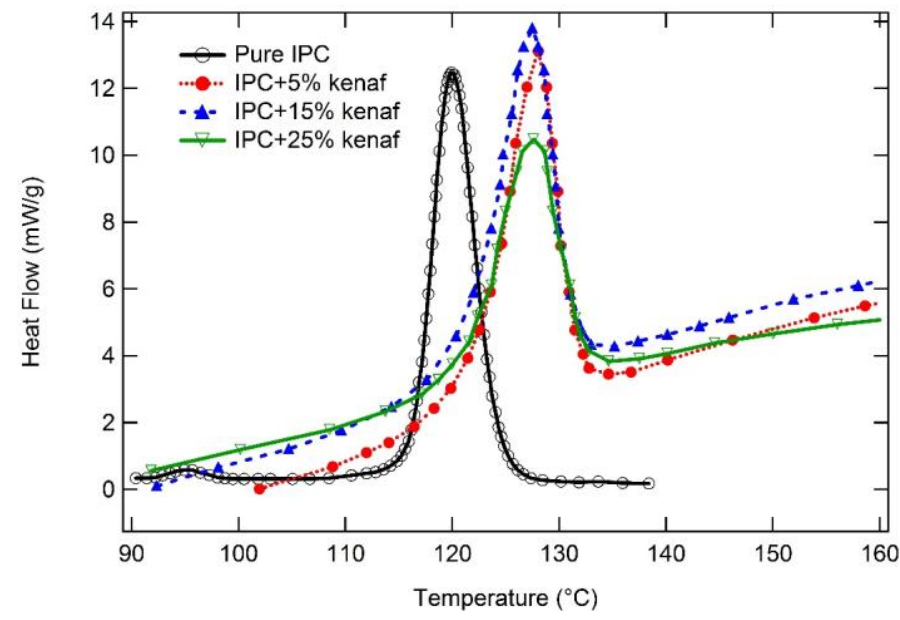

Figure 3 Melt crystallization exothermal curves for Pure IPC, IPC+ 5 wt.\% kenaf, IPC+ 15 wt.\% kenaf and IPC+ 25 wt.\% kenaf 
The data shown in Figure 3 were then used to calculate the relative crystallinity $\theta(\mathrm{t})$ by employing Equation 2. The results are given in Figure 2, which demonstrates relative crystallinity $\theta(\mathrm{t})$ as a function of crystallization time for pure IPC, IPC+ $5 \mathrm{wt} . \%$ kenaf, IPC+ 15 wt. $\%$ kenaf and IPC +25 wt. $\%$ kenaf. As shown in Figure 2, the addition of 5 wt. $\%$ kenaf yields faster crystallization kinetics (crystallization rate) in comparison with pure IPC. However, as kenaf concentration was increased, crystallization kinetics decreased rather than increased, as was our expectation. The explanation of this phenomenon is not yet clear. Generally, the greater the amount of crystallization seed (in this case kenaf fiber), the greater the amount of crystallization domain. This means that macroscopically, the crystallization kinetics in the form of crystallization time is higher, though microscopically the crystallization kinetics should be similar, due to its independence towards the number of crystallization seed. It can be suggested that high concentration of kenaf yields a high number of agglomeration of kenaf itself, thus preventing higher number of crystallization seeds.

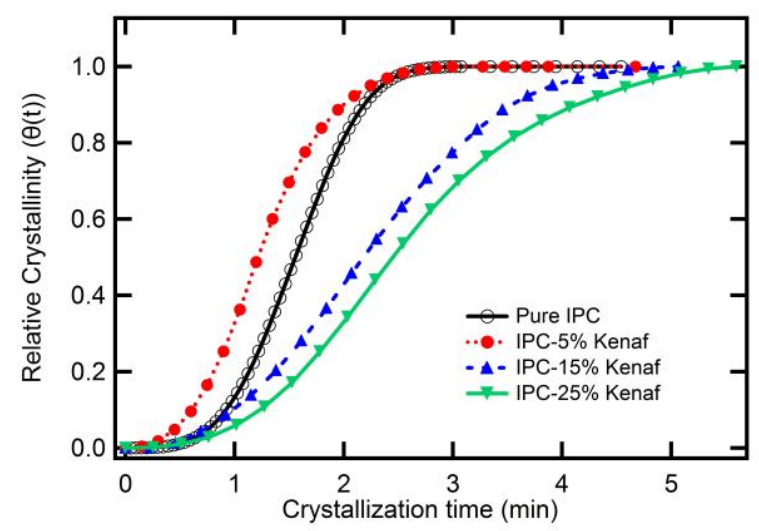

Figure 4 Plot of relative crystallinity for pure IPC, IPC+ 5 wt. $\%$ kenaf, IPC+ 15 wt.\% kenaf and IPC+ 25 wt.\% kenaf

Next the half-time parameter (time needed to achieve 50\% of maximum relative crystallinity) is discussed. The half-time of IPC and IPC+ kenaf is given in Figure 2. The half-time can be obtained by rearranging Equation 2 to obtain the following equation:

$$
t_{1 / 2}=\left(\frac{\ln (2)}{K_{t}(T)}\right)^{1 / n} .
$$

The calculation results demonstrated that as kenaf was added at $5 \mathrm{wt} . \%$ into IPC, the half-time significantly decreased from $93 \mathrm{~s}$ to $73 \mathrm{~s}$. This improvement of half-time will greatly affect cycle time in the manufacturing process of the polymer. However, as kenaf was increased to 15 wt. $\%$ and $25 \mathrm{wt} . \%$, the half-time increased even more than without any nucleating agent or the addition of kenaf (Figure 5). The explanation of this phenomenon is similar to our explanation for the slower crystallization rate at kenaf concentration of $15 \mathrm{wt} . \%$ and $25 \mathrm{wt} . \%$.

Next, the morphology of crystal growth through Avrami index $n$ is discussed. Avrami index and Avrami crystallization kinetics can be calculated by employing equation 2 . The curves obtained from Equation 2 were plotted in Figure 6. From curves obtained and plotted in Figure 6, one can determine the Avrami index and Avrami crystallization kinetics. In the Avrami model, the ideal system should follow the linear curve, especially for metal and pure polymer. However, with the addition of kenaf, one can expect that the linear curve deviates from linear behavior. This might be due to the inhomogeneous crystallization kinetics phenomenon which takes place. 


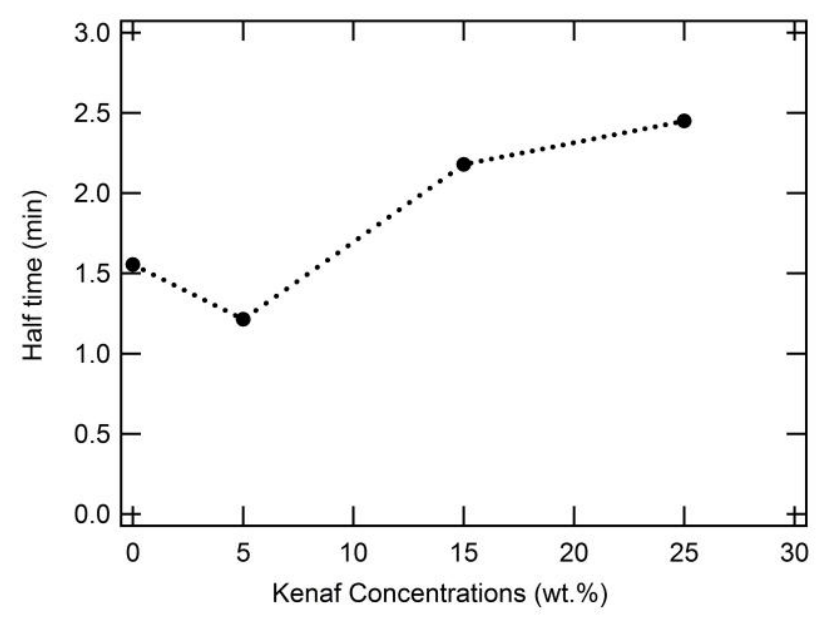

Figure 5 The half-time $\left(t_{1 / 2}\right)$ for pure IPC, IPC+ 5 wt. $\%$ kenaf, IPC+ 15 wt. $\%$ kenaf and IPC+ 25 wt. $\%$ kenaf

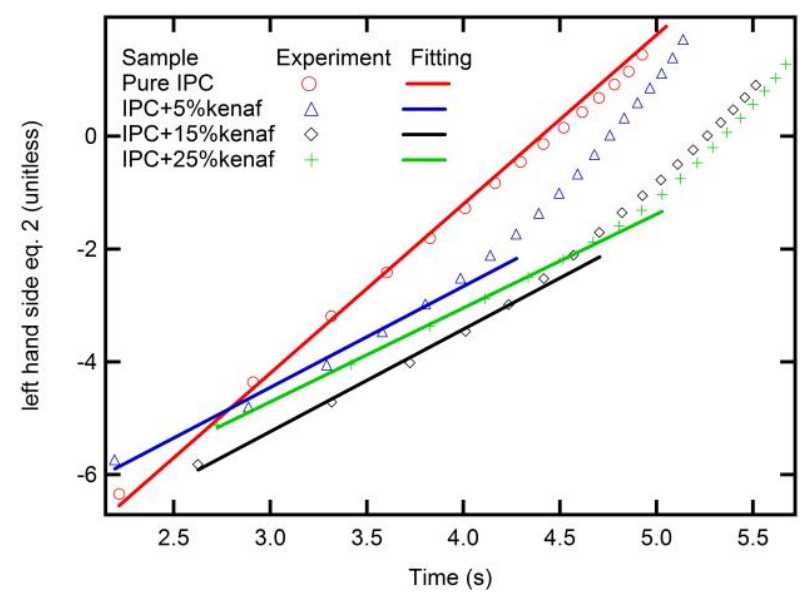

Figure 6 The plot of $\ln [-\ln (1-\theta(t))]$ vs time for pure IPC, IPC+ $5 \mathrm{wt} . \%$ kenaf, IPC $+15 \mathrm{wt} . \%$ kenaf and IPC $+25 \mathrm{wt} . \%$ kenaf. For the pure IPC, the Avrami model shows only linear curve from the beginning until the final state of crystallization. However, as kenaf in the different concentration were added, the linearity only holds at the beginning stage of crystallization

Actually, the crystallization kinetics discussion in iPP/IPC should incorporate the existence of $\alpha$ and $\beta$ forms of iPP (Nakamura et al., 2008). However, in this study only general crystallization kinetics were considered without differentiating between $\alpha$ and $\beta$ forms. Though, the hint about the existence of different type of crystallization kinetics is clearly observed from Figure 6 . In the next paragraph, this phenomenon will be discussed in depth.

For the pure IPC, the Avrami model shows only one type of crystallization kinetics, as demonstrated by linear curve from the beginning until the final state of crystallization. However, as kenaf in the various concentrations was added, the linearity only holds at the beginning stage of crystallization. The linearity holds only for $4.2 \mathrm{mins}, 4.6 \mathrm{mins}$ and $4.4 \mathrm{mins}$ for IPC+ $5 \mathrm{wt} . \%$ kenaf, IPC+ $10 \mathrm{wt} . \%$ kenaf and IPC+ $15 \mathrm{wt} \%$ kenaf respectively. It can be recalled that the stage when the linearity holds as primary crystallization stage and other region as secondary crystallization stage. Secondary crystallization might be important when one considers that the heat transfer between kenaf and IPC should be taken into account. However, the study of heat transfer between nucleating agent and polymer matrix is scarce and complicated due to the complex behavior of heat transfer between those two materials. Clearly 
as shown in Figure 6 below, the secondary crystallization stage shows a different Avrami parameter (thus changing the physical parameter of growth), especially for the morphology parameter $n$ (Avrami index). Although it is too early to claim that this is due to secondary crystallinity, this experiment was conducted in non-equilibrium steady state condition. In the non-equilibrium steady state condition fascinating new effects may exist (Fatriansyah \& Orihara, 2015). All of the curves show a steeper curve for the secondary crystallization stage, which means that the $n$ increases. The increase of $n$ has the physical meaning that the crystal growth dimension changes from fiber-like crystal growth to the higher dimension crystal growth (this will be discussed further in the next paragraph). Higher dimension crystal growth should be avoided when considering the role of fiber as reinforcement. The tensile strength may decrease in that case.

Avrami indices $n$ for pure IPC, IPC+ 5 wt.\% kenaf, IPC+ 15 wt.\% kenaf and IPC+ 25 wt.\% kenaf were determined by calculating the gradient from the curves shown in Figure 6 and the values then were plotted in Figure 7. The addition of kenaf reduces the Avrami index. This means that the crystal growth changes from 3-dimensional crystal growth to the direction of 1 dimensional crystal growth. The decrease of the Avrami index was observed in all concentrations of kenaf which shows that the fiber of kenaf directs the crystal growth. There is a computer simulation which was conducted by Zhouzhou et al. (2018) that showed that the addition of a one dimensional object in polymer matrices induces the one-dimensional crystal growth which is preferable in reinforcement applications. Properly controlled direction of one dimensional crystal growth yields higher material strength in the direction of crystal growth. However, to properly control the direction of fiber in order to induce crystal growth in a specific direction is very difficult. Proper study in fiber direction in polymer melts is needed.

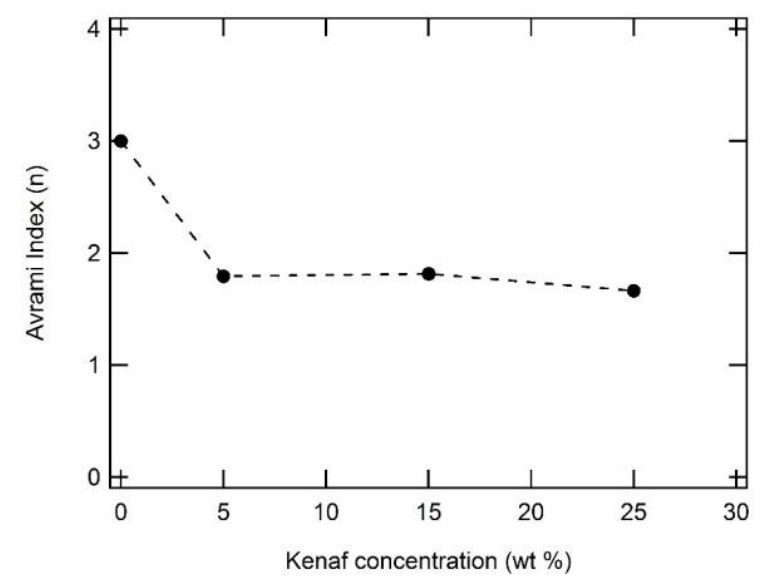

Figure 7 Avrami index $n$ for pure IPC, IPC+ 5 wt. $\%$ kenaf, IPC+ 15 wt. $\%$ kenaf and IPC+ 25 wt. $\%$ kenaf. It is shown that the addition of kenaf reduces the Avrami index

Avrami crystallization kinetics determined from Equation 2 and curves in Figure 6 were plotted in Figure 8. From Figure 8, it can be shown that the addition of kenaf yields a higher Avrami crystallization kinetics parameter. It should be noted that this value is obtained by employing the Avrami model and may not reflect real kinetics of the system. However, it is adequate to quantify the crystallization kinetics phenomenon. There is a mismatch between value of the Avrami crystallization kinetics and half-time. Generally, if Equations 1 to 3 were properly observed, one can observe that higher Avrami crystallization kinetics reflects a lower half-time. For concentration of kenaf $5 \mathrm{wt} . \%$, the statement is valid. However, in higher concentration of kenaf (15 wt.\% and 25 wt.\%), this is not the case. This might be due to the existence of a 
secondary crystallization phenomenon (see Figure 6) which induces complex relationship between crystallization kinetics and half-time.

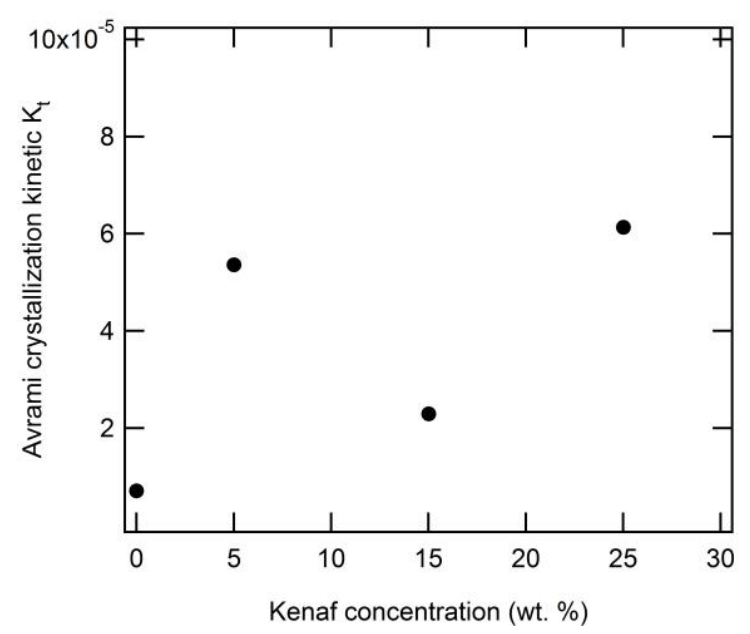

Figure 8 Avrami crystallization kinetic $K_{t}$ for pure IPC, IPC+ 5 wt. $\%$ kenaf, IPC+ 15 wt. $\%$ kenaf and IPC+ 25 wt.\% kenaf. The addition of kenaf yields higher Avrami crystallization kinetics

Next, the connection between the crystallization kinetics phenomenon (crystal growth, not to be confused with Avrami crystallization kinetic $K_{t}$ ) with the strength of materials is discussed. The main purpose of the addition of the kenaf fiber in this paper is to improve crystallization kinetics (kenaf as a nucleating agent). The other additional purpose is to utilize kenaf fiber as a reinforcement. However, the use of kenaf fiber as a reinforcement needs proper orientation of kenaf fiber inside IPC. The homogenous direction (unidirectional) of the kenaf fiber is important to increase tensile strength in the preferred direction (which is similar to kenaf fiber orientation/direction). In our research, the direction of kenaf fiber cannot be controlled. Nonetheless, despite the limitation of being unable to control direction, the connection between the crystallization kinetics parameter and the strength of the materials (through tensile strength) should be discussed anyway.

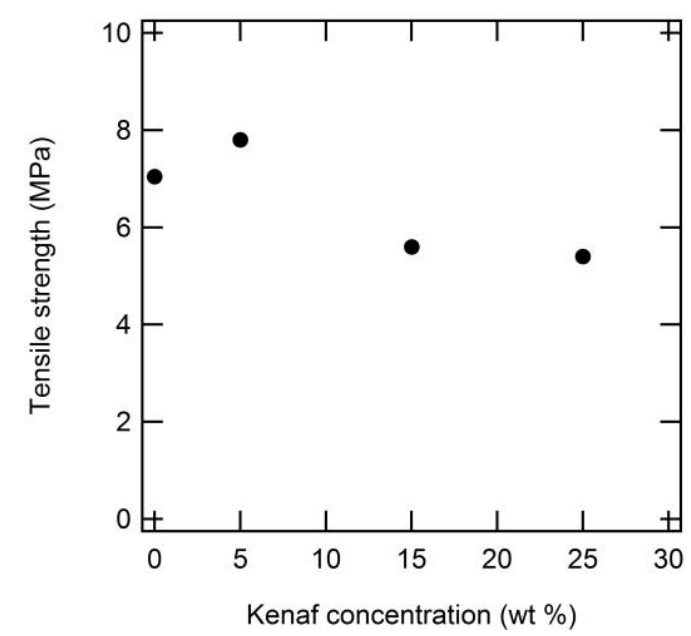

Figure 9 Tensile strength of IPC, IPC+ 5 wt. $\%$ kenaf, IPC+ 15 wt. $\%$ kenaf and IPC+ 25 wt. $\%$ kenaf

As shown in Figure 9, the addition of kenaf of $5 \mathrm{wt} . \%$ concentration increases tensile strength. However, as kenaf concentration was increased, the tensile strength decreased. This tensile strength results are in agreement with the crystallization kinetics results. The addition of $5 \mathrm{wt} . \%$ 
of kenaf concentration is improving both crystallization kinetics and tensile strength (mechanical properties) and furthermore the addition of kenaf is lowering crystallization kinetics and tensile strength (mechanical properties). This demonstrates that there is a connection between crystallization kinetics and the strength of materials. The higher crystallization kinetics induces more homogenous and more unidirectional crystal growth, thus improving the strength of the materials. It can be suggested that the addition of more than 5 wt.\% kenaf concentration may lead to agglomeration of kenaf fiber, thus lowering crystallization kinetics and tensile strength (mechanical properties).

\section{CONCLUSION}

The alkalization of kenaf by $\mathrm{NaOH}$ was partially successful at removing dirt on the surface of kenaf and reduced lignin, which was investigated by means of FESEM and FTIR. The reduction of lignin decreased the polarity of kenaf and made it more compatible with IPC. The addition of kenaf at concentration of $5 \mathrm{wt} \%$ increased the crystallization kinetics in the following ways: rate of crystallization (crystallization time) parameter, Avrami crystallization kinetic parameter and reduced Avrami index. In general, the addition of kenaf at concentration of 5 wt. \% improves crystal growth dynamics and morphology (crystal growth in one dimension). However, further addition of kenaf does not improve growth dynamics and morphology. This phenomenon could be caused by agglomeration and thus preventing effective heat transfer between crystal seeds and the matrix. In addition, the connection between crystal growth dynamics and mechanical strength (tensile strength) has been successfully established through crystallization kinetics and mechanical test results. It is shown that better crystal growth dynamics yields better mechanical strength in the IPC+ kenaf system.

\section{ACKNOWLEDGEMENT}

This work was supported by Universitas Indonesia through HIBAH PITTA A 2019 under contract number NKB-0464/UN2.R3.1/HKP.05.00/2019.

\section{REFERENCES}

Abral, H., Kasmianto, E., Mastariyanto, P., 2012. Mechanical Properties and Microstructure of Metroxylon Sago Fiber Treated by Sodium Hydroxide. International Journal of Technology, Volume 3(1), pp. 16-23

Akhtar, M.N., Sulong, A.B., Fadzly Radzi, M.K.F., Ismail, N.F., Raza, M.R., Muhamad, N., Khan, M.A., 2016. Influence of Alkaline Treatment and Fiber Loading on the Physical and Mechanical Properties of Kenaf/Polypropylene Composites for Variety of Applications. Progress in Natural Science: Materials International, Volume 26(6), pp. 657-664

Akil, H., Omar, M.F., Mazuki, A.A.M., Safiee, S.Z.A.M., Ishak, Z.M., Bakar, A.A., 2011. Kenaf Fiber Reinforced Composites: A Review. Materials \& Design, Volume 32(8-9), pp. 4107-4121

Avrami, M., 1939. Kinetics of Phase Change. I: General Theory. Journal of Chemical Physics, Volume 7, pp. 1103-1112

Chalid, M., Prabowo, I., 2015. The Effects of Alkalization to the Mechanical Properties of the Ijuk Fiber Reinforced PLA Biocomposites. International Journal of Chemical and Molecular Engineering, Volume 9(2), pp. 342-346

Chalid, M., Fikri, A.I., Satrio, H.H., Fatriansyah, J.F., 2017. An Investigation of the Melting Temperature Effect on the Rate of Solidification in Polymer using a Modified Phase Field Model. International Journal of Technology, Volume 8(7), pp. 1321-1328 
Chalid, M., Fikri, A.I., Gregory, N., Priadi, D., Fatriansyah, J.F., 2018. Study of Crystallization Kinetics of Peek Thermoplastics using Nakamura Equation. In: AIP Conference Proceedings, Volume 1945(1)

Chen, Y.H., Zhong, G.J., Wang, Y., Li, Z.M., Li, L., 2009. Unusual Tuning of Mechanical Properties of Isotactic Polypropylene using Counteraction of Shear Flow and B-Nucleating Agent on B-Form Nucleation. Macromolecules, Volume 42(12), pp. 4343-4348

Fatriansyah, J.F., Orihara, H., 2015. Electric-field-induced Flow-aligning state in a Nematic Liquid Crystal. Physical Review E, Statistical, Nonlinear, and Soft Matter Physics, Volume 91(4), pp. 042508-1-042508-7

Fundador, N.G.V., Enomoto-Rogers, Y., Takemura, A., Iwata, T., 2012. Syntheses and Characterization of Xylan Esters. Polymer, Volume 53(18), pp. 3885-3893

Guo, L., Chen, F., Zhou, Y., Liu, X., Xu, W., 2015. The Influence of Interface and Thermal Conductivity of Filler on the Nonisothermal Crystallization Kinetics of Polypropylene/Natural Protein Fiber Composites. Composites Part B: Engineering, Volume 68, pp. 300-309

Hongjun, C., Xiaolie, L., Dezhu, M., Jianmin, W., Hongsheng, T., 1999. Structure and Properties of Impact Copolymer Polypropylene. I. Chain Structure. Journal of Applied Polymer Science, Volume 71(1), pp. 93-101

Karger-Kocsis, J., Varga, J., Ehrenstein, G.W., 1997. Comparison of The Fracture and Failure Behavior of Injection- Molded A- And B- Polypropylene in High- Speed Three- Point Bending Tests. Journal of Applied Polymer Science, Volume 64(11), pp. 2057-2066

Mahadevan, S., Giridhar, A., Singh, A.K., 1986. Calorimetric Measurements on As-Sb-Se Glasses. Journal of Non-Crystalline Solids, Volume 88(1), pp. 11-34

Michael, L., 1991, SPI Plastics Engineering Handbook Society of the Plastics Industry. New York: Inc. Springer US

Nakamura, K., Shimizu, S., Umemoto, S., Thierry, A., Lotz, B., \& Okui, N., 2008. Temperature dependence of crystal growth rate for $\alpha$ and $\beta$ forms of isotactic polypropylene. Polymer journal, 40(9), pp. 915.

Prabowo, I., Pratama, J.N., Chalid, M., 2017. The Effect of Modified Ijuk Fibers to Crystallinity of Polypropylene Composite. In: IOP Conference Series: Materials Science and Engineering, Volume 223

Spoljaric, S., Genovese, A., Shanks, R.A., 2009. Polypropylene-Microcrystalline Cellulose Composites with Enhanced Compatibility and Properties. Composites Part A: Applied Science and Manufacturing, Volume 40(6-7), 791-799

Tolinski, M., 2009. Getting the Most Out of Polypropylene, Polyethylene and TPO. $2^{\text {nd }}$ Edition Additives for Polyolefins, Elsevier Inc., London

Yuanita, E., Pratama, J.N., Mustafa, J.H., Chalid, M., 2015. Multistages Preparation for Microfibrillated Celluloses based on Arenga Pinnata "Ijuk" Fiber. Procedia Chemistry, Volume 16, pp. 608-615

Zhouzhou G., Rui Y., Jun Y., Xiaoyan Q., Rongjuan L., Yong L., Zhiping Z., Yijing N., 2018. Dynamic Monte Carlo Simulations of Effects of Nanoparticle on Polymer Crystallization in Polymer Solutions. Computational Materials Science, Volume 147, pp. 217-226 